Case Report

\title{
Histomorphologic and Immunohistochemical Characterization of a Cardiac Purkinjeoma in a Bearded Seal (Erignathus barbatus)
}

\author{
G. Krafsur, ${ }^{1,2}$ E. J. Ehrhart, ${ }^{1}$ J. Ramos-Vara, ${ }^{3}$ G. Mason, ${ }^{1}$ F. Sarren, ${ }^{2}$ \\ B. Adams, ${ }^{2}$ C. Hanns, ${ }^{2}$ T. Spraker, ${ }^{1}$ and C. Duncan ${ }^{1,4}$ \\ ${ }^{1}$ CSU Veterinary Diagnostic Medicine Center, Fort Collins, CO, USA \\ ${ }^{2}$ North Slope Borough Department of Wildlife Management, Barrow, AK, USA \\ ${ }^{3}$ Indiana Animal Disease Diagnostic Laboratory and Department of Comparative Pathobiology, Purdue University, \\ West Lafayette, IN, USA \\ ${ }^{4}$ Colorado State University Veterinary Diagnostic Laboratory, 300 West Drake Avenue, Fort Collins, CO 80526, USA
}

Correspondence should be addressed to C. Duncan; colleen.duncan@colostate.edu

Received 30 May 2014; Accepted 13 August 2014; Published 31 August 2014

Academic Editor: Luciano Espino López

Copyright (C) 2014 G. Krafsur et al. This is an open access article distributed under the Creative Commons Attribution License, which permits unrestricted use, distribution, and reproduction in any medium, provided the original work is properly cited.

\begin{abstract}
The most common cardiac tumors of heart muscle are rhabdomyomas, solitary or multiple benign tumors of striated muscle origin. While cardiac rhabdomyomas are well described in human medical literature, limited information depicting the occurrence of cardiac rhabdomyomas in veterinary species exists. A case of multiple firm white nonencapsulated nodules in the heart of a bearded seal is described. Microscopic findings included cytoplasmic vacuolization with formation of spider cells, glycogen vacuoles, and striated myofibrils. These cells expressed immunoreactivity for neuron-specific enolase and protein gene product 9.5, a marker for neuronal tissue and Purkinje fiber cells. Immunoreactivity for protein gene product 9.5 along with other microscopic findings substantiates Purkinje fiber cell origin of the cardiac rhabdomyoma in the bearded seal and use of the term purkinjeoma to describe this lesion.
\end{abstract}

\section{Introduction}

Rhabdomyomas, benign tumors of striated muscle origin, most commonly occur in the heart and are the most frequently diagnosed primary cardiac tumor of infants and children [1-3]. Multiple cardiac rhabdomyomas are frequently indicative of tuberous sclerosis, an autosomal dominant condition with an array of clinical manifestations, most notably seizures, cognitive deficits, behavioral disturbances, and adenoma sebaceum $[1,3]$. Additional lesions associated with tuberous sclerosis include cortical hamartomas, giant cell astrocytomas, pulmonary lymphangiomyomatosis, and renal angiomyolipomas [3]. Cardiac rhabdomyomas may be clinically silent with spontaneous regression in utero or during early childhood being the norm [1-3]. In veterinary medicine, cardiac rhabdomyomas are most commonly identified as incidental lesions in domesticated pigs [4-6] with rare occurrence documented in sheep [4], dogs [7, 8], and deer [9]. The occurrence of cardiac rhabdomyomas in pigs appears to be breed associated, with a higher incidence of the tumors occurring in red wattle and red wattle-crossbred piglets while similar cardiac rhabdomyomas were rare in other pig breeds submitted to the same diagnostic laboratory [9].

The typical macroscopic appearance of cardiac rhabdomyomas is white to tan, single or multiple, and nodular nonencapsulated masses most commonly occurring in the left ventricular wall and to a lesser extent in the right ventricle, right atrium, interventricular septum, and the main pulmonary artery [2]. Tumor nodules are composed of pleomorphic, vacuolated cells with or without glycogen 
[4]. Postmortem glycolysis with delayed formalin fixation or routine histologic processing with subsequent removal of glycogen results in the distinctive appearance of spider cells with a central nucleus suspended in a network of cross striated myofibrils; however positive periodic acid-Schiff (PAS) staining confirms glycogen content [4].

Additional phosphotungsten acid-hematoxylin staining (PTAH) permits enhanced visualization of myofibrils with cross striations $[4,6,9]$. Large nuclei with stippled chromatin, prominent nucleoli, and binucleate cells are observed. Mitotic figures are usually not appreciated [6-9].

The nature and histogenesis of cardiac rhabdomyomas remains unclear. Cardiac rhabdomyomas exhibit immunoreactivity with myoglobin, desmin, vimentin, neuron-specific enolase (NSE), human natural killer cell-1(HNK-1/Leu-7), and atrial natriuretic peptide (ANP) markers, similar to normal cardiomyocytes $[5,10]$. Protein gene product 9.5 (PGP 9.5), also known as ubiquitin carboxyl-terminal hydrolase1 , is frequently expressed by neuronal cells and serves as a reliable marker for Purkinje fiber cells [10]. Immunoreactivity for PGP 9.5 has been demonstrated in porcine cardiac rhabdomyomas, supporting Purkinje fiber cell origins of cardiac rhabdomyomas [5]. This report describes multiple cardiac rhabdomyomas in a bearded seal (Erignathus barbatus) with gross, histopathological, and immunohistochemical (IHC) findings similar to those seen in cardiac rhabdomyomas in other species and supportive of Purkinje fiber cell origin.

\section{Case Presentation}

In July 2010, as part of a long term collaborative study between Iñupiat eskimo subsistence hunters and The North Slope Borough Department of Wildlife Management, abdominal and thoracic viscera from a one-year-old female bearded seal were submitted for gross examination and diagnostic sampling. Greater than 15 discrete nodular masses ranging in size from 0.5 to $2.0 \mathrm{~cm}$ were present in all four chambers of the heart (Figure 1) none of which protruded into the lumen. No gross changes related to heart failure were appreciated in the heart, lungs, liver, or spleen. The pale white, variably sized nonencapsulated masses were smooth, firm, and homogenous. The masses were well demarcated from adjacent normal cardiovascular stroma and did not bulge on cut surface. Representative tissue samples from the heart, skin, thyroid and adrenal gland, lymph node, skeletal muscle, ovary, lung, spleen, liver, pancreas, stomach, and intestine were fixed in $10 \%$ buffered formalin, routinely processed, and stained with H\&E, PAS, PTAH, desmin, and vimentin. To detect neuronal and Purkinje fiber cell markers, immunohistochemistry (IHC) was performed with standard indirect technique utilizing a secondary antibody conjugated to a HRP labeled polymer (DakoEnVision+, Dakocytomation) and DAB chromogen with hematoxylin counterstain. Primary antibodies used were PGP 9.5 rabbit polyclonal antibody (Z5116, Dako, Carpinteria) at 1:50 dilution and NSE mouse monoclonal antibody (M0873 clone BBS/NC/VIH14, Dako) at 1:200 dilution and 60 min room temperature incubation. Antigen retrieval using a decloaker chamber with

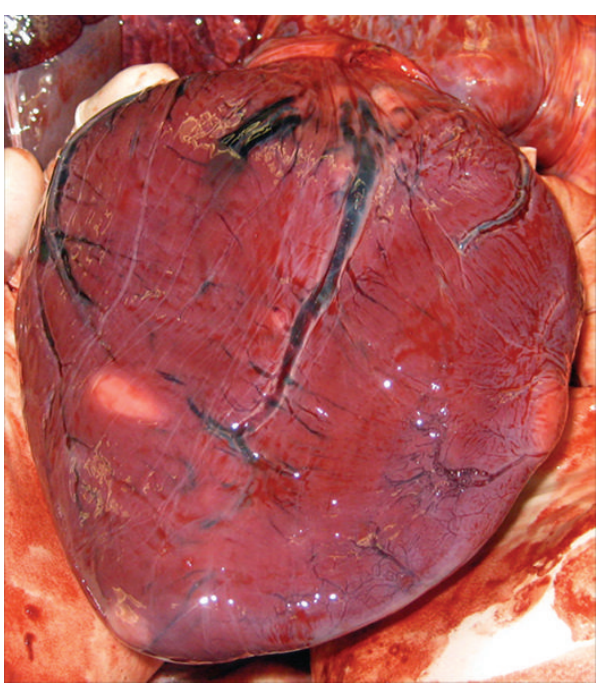

FIgURE 1: Heart; bearded seal. Multiple pale white discrete nodular masses expand the cardiac muscle (purkinjeomas).

pH 7.6 citrate buffer for NSE staining was performed prior to antibody incubation.

Histologically, the masses were nonencapsulated and variably demarcated from normal myocardium but occasionally infiltrated between cardiomyocytes as streaming bundles. Cells were polygonal with inconspicuous borders and had abundant eosinophilic cytoplasm which contained variably sized vacuoles (Figure 2). Some vacuolated cells exhibited a "spider cell" appearance characterized by a central cytoplasmic mass containing the nucleus suspended in a network of striated myofibrils (Figure 2, arrow). The nuclei were round to oval with clumped chromatin and prominent nucleoli. Anisocytosis, anisokaryosis, and occasional binucleate cells were present. Mitotic figures were not appreciated. A small focus of lymphoplasmacytic infiltration was observed in one of the masses. Neoplastic cells had PAS positive material within cytoplasmic vacuoles and within cytoplasmic projections (Figure 3); adjacent normal cardiomyocytes were PAS negative. Distinct striated myofibrils were present in PTAH stained sections of normal cardiomyocytes, but infrequent in neoplastic cells (Figure 4). Both the atypical cells and the adjacent normal myocardial cells stained similarly for desmin and vimentin. Moderate, diffuse cytoplasmic expression for PGP9.5 was present in some of the tumor cells while expression in adjacent normal cardiomyocytes was weak to absent (Figure 5). Immunoreactivity for neuron-specific enolase (NSE) was rare in the atypical cells (Figure 6).

Additional histologic lesions also present in this animal were incidental changes commonly seen in hunter harvested animals of this species (G. Krafsur, T. Spraker, unpublished personal observations) including mild exertional rhabdomyolysis with rare myositis, pulmonary nematodiasis characterized by Parafilaroides spp. in the alveolar spaces and airways, and occasional lymphoplasmacytic infiltrates throughout the hepatic parenchyma most likely associated with migration tracts of Orthosplanchnus arcticus. 


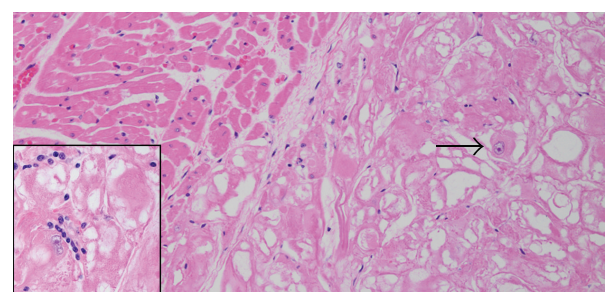

FIGURE 2: Heart; bearded seal. Neoplastic cells are large and vacuolated and occasional spider cells are also observed (arrow) and rare foci of lymphocytes are present (insert). H\&E.

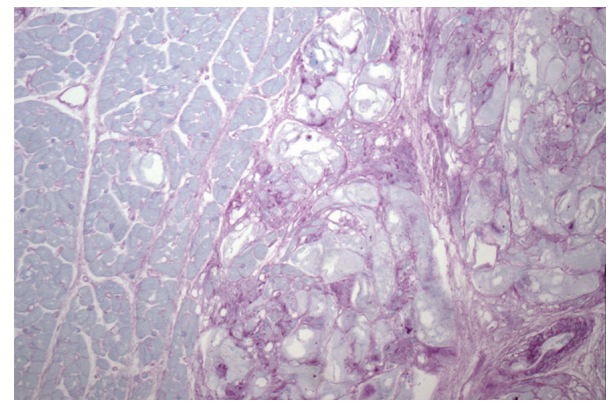

FIGURE 3: Heart; bearded seal. PAS positive granular material consistent with glycogen is present in the cytoplasm of neoplastic but not normal myocardium. PAS.

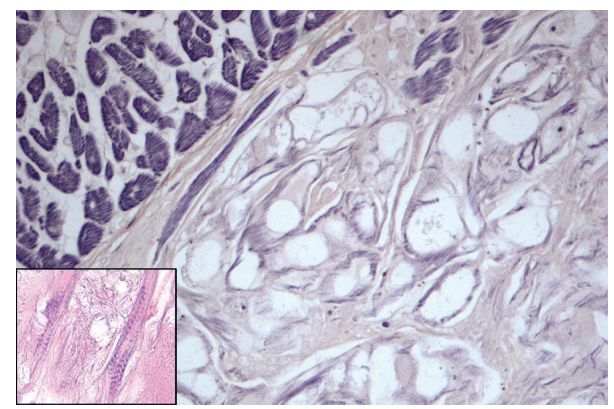

FIgURE 4: Heart; bearded seal. Normal myocardium and some rhabdomyoma cells are positive for PTAH. Striations (insert) are more common in normal cardiomyocytes but are occasionally observed in neoplastic cells. PTAH.

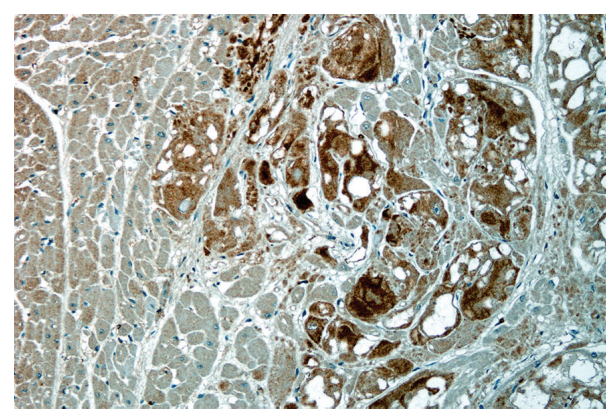

FIGURE 5: Heart; bearded seal. Neoplastic cells are moderately immunoreactive for protein gene product 9.5.

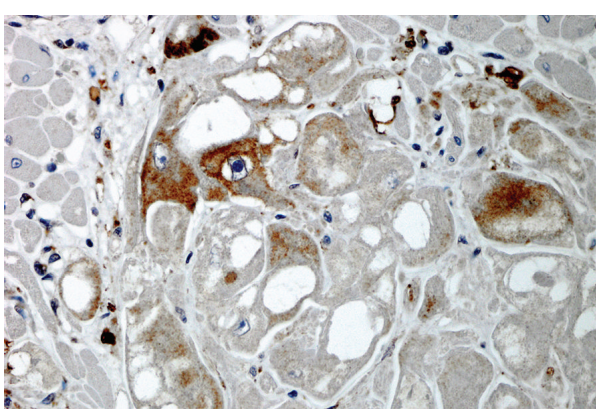

Figure 6: Heart; bearded seal. Neoplastic cells exhibit weak cytoplasmic staining for neuron-specific enolase.

\section{Discussion}

Limited scientific literature exists describing the incidence of cardiac rhabdomyomas in veterinary species. The veterinary literature contains only two cases describing clinical manifestations associated with cardiac rhabdomyomas including agonal convulsions with sudden death in a captive fallow deer and chylopericardium and right-sided congestive heart failure in a Staffordshire Bull Terrier that were consistent with cardiac failure likely from outflow obstruction and conduction disturbances $[7,9]$. While the presence of multiple variably sized cardiac rhabdomyomas in the bearded seal heart did not appear to cause overt signs of heart failure, the numerous lesions in all four chambers of the heart may have resulted in aberrations of impulse conduction or subclinical arrhythmias.

Considerable debate exists regarding the nature and histogenesis of cardiac rhabdomyomas. Ultrastructural studies and the use of markers such as NSE and PGP 9.5 have been used to gain insight regarding the cells of origin and whether they derive from striated myocardium or Purkinje fiber cells. Purkinje fibers, located in the subendocardial and ventricular myocardium, are the conducting fibers of the heart that permit coordinated contraction of the ventricles. Purkinje fibers are neither neurons nor nerve cells but rather specialized myocytes arranged in aggregates or fibers. Larger than cardiomyocytes, Purkinje fibers are not contractile and lack a sarcoplasmic reticulum, $t$-tubules, and intercalated discs, features typical of cardiomyocytes [2]. The microscopic appearance of Purkinje fiber cells is different from cardiomyocytes; Purkinje fiber cells are larger, with scant myofibrils, and abundant glycogen resulting in less intense eosin staining than adjacent myocardium $[2,4]$. The histogenesis of cardiac rhabdomyomas is complicated with ultrastructural characteristics indicative of both cardiomyocyte and Purkinje fiber cell origins reported in studies of human cardiac rhabdomyomas [2]. Intercalated discs, specialized cell-cell attachments between adjacent cardiomyocytes, were identified in one of the rhabdomyomas while desmosomal attachments, typical for Purkinje fiber cells, were visualized in the remaining two rhabdomyomas [2]. Microscopically, nodules from the bearded seal cardiac rhabdomyomas were composed of a preponderance of large, polygonal vacuolated cells with a paucity of striated myofibrils, characteristic features of 
Purkinje fiber cells. PAS stains did demonstrate the presence of a granular material consistent with glycogen suggesting the rhabdomyoma cells are of a Purkinje fiber cell origin. Sparsely distributed myofibrils were appreciated within the vacuolated cells from identical sections of PTAH stained bearded seal cardiac rhabdomyoma cells. The myofibrils were microscopically indistinguishable from those in adjacent normal myocardium. The paucity of cross striations in PTAH stained sections of bearded seal cardiac rhabdomyoma cells offers further evidence that these cells derive from Purkinjefiber cells. Studies investigating cardiac innervation and the conduction system of the fetal and adult camelid heart have shown that PGP 9.5 is a reliable marker for the identification of Purkinje fiber cells [10]. These findings along with strong PGP 9.5 immunoreactivity appreciated in swine cardiac rhabdomyomas support the use of PGP 9.5 as a reliable marker for the identification of Purkinje fiber cells $[5,10]$.

This report provides the first description of multiple cardiac rhabdomyomas in a bearded seal. The enlarged pleomorphic vacuolated spider cells, glycogen positive staining, limited striated myofibrils, and immunoreactivity for Purkinje fiber markers NSE and PGP 9.5 strongly characterize the Purkinje fiber origin of these cardiac rhabdomyomas and support the use of purkinjeoma as a descriptor for this tumor.

\section{Conflict of Interests}

The authors declare that there is no conflict of interests regarding the publication of this paper.

\section{Acknowledgments}

G. Krafsur wishes to thank Jill-Marie Seymour and Sara Carroll, graduate students from the University of AlaskaFairbanks School of Fisheries and Ocean Sciences, for their assistance with the collection of tissues. Thanks are due to Lara Horstmann-Dehn, professor in the School of Fisheries and Ocean Science at the University of Alaska-Fairbanks, for her expertise in aging the bearded seal. Thanks are due to Barrow subsistence hunter Felton Sarren for allowing the lead investigator to examine and collect tissues from the subsistence harvested bearded seal. Finally, the authors are deeply indebted to Billy Adams and the rest of the scientists and staff at the North Slope Borough Department of Wildlife Management for their technical assistance and generous financial support of this work. Financial support for the costs associated with tissue processing and slide preparation was provided by the North Slope Borough Department of Wildlife Management, Barrow, Alaska.

\section{References}

[1] A. Nir, A. J. Tajik, W. K. Freeman et al., "Tuberous sclerosis and cardiac rhabdomyoma," The American Journal of Cardiology, vol. 76, no. 5, pp. 419-421, 1995.

[2] J. F. Silverman, S. Kay, C. M. McCue, R. R. Lower, A. J. Brough, and C. H. Chang, "Rhabdomyoma of the heart: ultrastructural study of three cases," Laboratory Investigation, vol. 35, no. 6, pp. 596-606, 1976.
[3] D. M. Weiner, D. H. Ewalt, E. S. Roach, and T. W. Hensle, "The tuberous sclerosis complex: a comprehensive review," Journal of the American College of Surgeons, vol. 187, no. 5, pp. 548-561, 1998.

[4] R. Bradley, G. A. H. Wells, and J. B. R. Arbuckle, "Ovine and porcine so-called cardiac rhabdomyoma (Hamartoma)," Journal of Comparative Pathology, vol. 90, no. 4, pp. 551-558, 1980.

[5] B. Jacobsen, M. Kreutzer, D. Meemken, W. Baumgärtner, and C. Herden, "Proposing the term purkinjeoma: protein gene product 9.5 expression in 2 porcine cardiac rhabdomyomas indicates possible purkinje fiber cell origin," Veterinary Pathology, vol. 47, no. 4, pp. 738-740, 2010.

[6] B. J. McEwen, "Congenital cardiac rhabdomyomas in red wattle pigs," Canadian Veterinary Journal, vol. 35, no. 1, pp. 48-49, 1994.

[7] C. S. Mansfield, J. J. Callanan, and H. McAllister, "Intraatrial rhabdomyoma causing chylopericardium and right-sided congestive heart failure in a dog," Veterinary Record, vol. 147, no. 10, pp. 264-267, 2000.

[8] Z. A. Radi and A. Metz, "Canine cardiac rhabdomyoma," Toxicologic Pathology, vol. 37, no. 3, pp. 348-350, 2009.

[9] C. Kolly, A. Bidaut, and N. Robert, "Cardiac rhabdomyoma in a juvenile fallow deer (Dama dama)," Journal of Wildlife Diseases, vol. 40, no. 3, pp. 603-606, 2004.

[10] A. A. El Sharaby, M. Egerbacher, A. K. Hammoda, and P. Böck, "Immunohistochemical demonstration of Leu-7 (HNK-1), Neurone-Specific Enolase (NSE) and Protein-Gene Peptide (PGP) 9.5 in the developing camel (Camelus dromedarius) heart," Anatomia, Histologia, Embryologia, vol. 30, no. 6, pp. 321-325, 2001. 

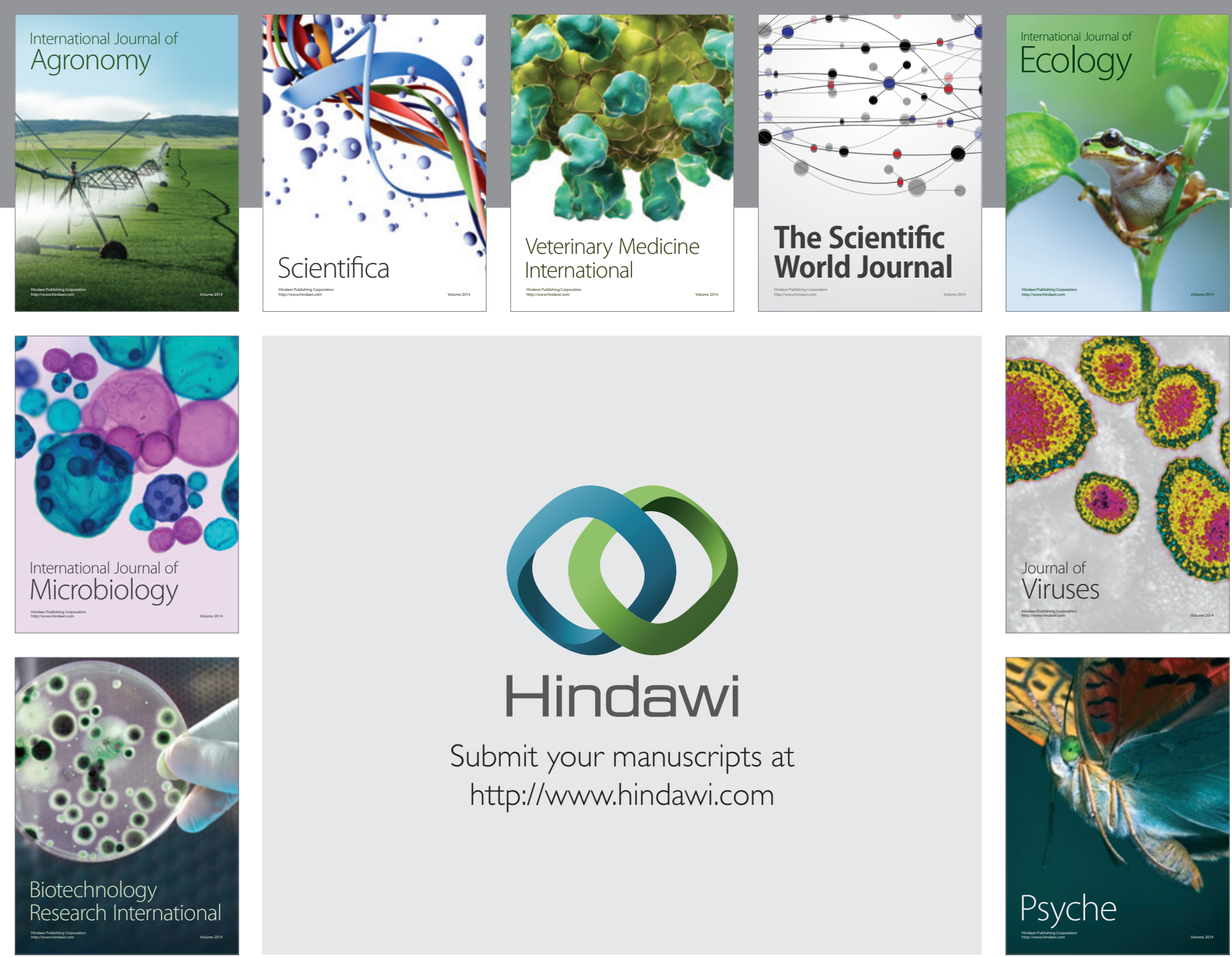

Submit your manuscripts at http://www.hindawi.com
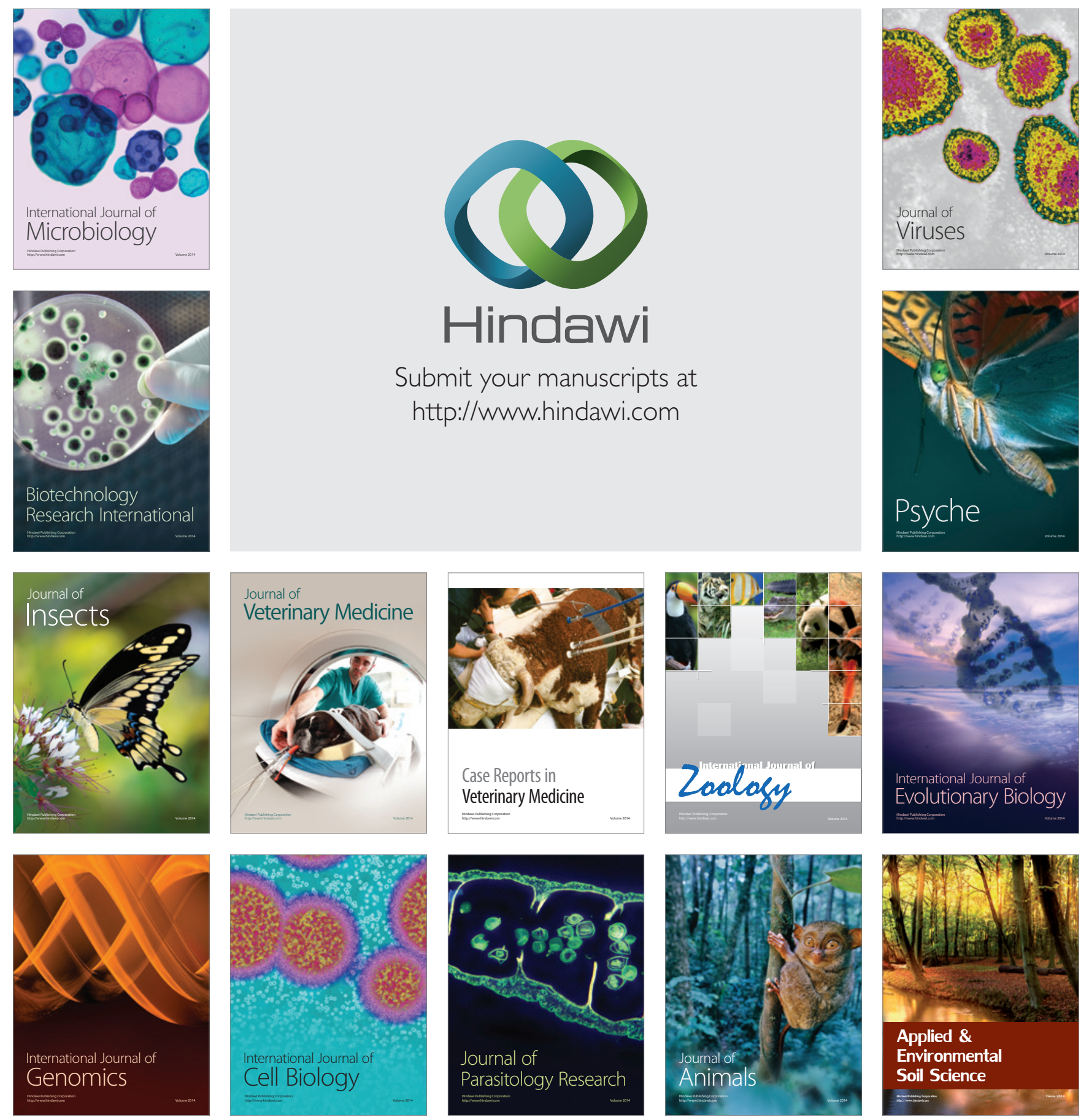